\title{
Better Understanding of Direct Bone-Conduction Measurement: Comparison with Frequency-Specific Bone-Conduction Tones and Brainstem Responses
}

\author{
Yeoju Kim ${ }^{1,2}$, Woojae Han ${ }^{1,2,3}$, Sihun Park ${ }^{1,2}$, Sunghwa You, ${ }^{1,2}$, \\ Chanbeom Kwak ${ }^{1,2}$, Youngjoon Seo ${ }^{4}$, and Jihyeon Lee ${ }^{1,4}$ \\ ${ }^{1}$ Laboratory of Hearing and Technology, ${ }^{2}$ Division of Speech Pathology and Audiology, \\ ${ }^{3}$ Research Institute of Audiology and Speech Pathology, College of Natural Sciences, Hallym University, Chuncheon, Korea \\ ${ }^{4}$ Department of Otorhinolaryngology, Yonsei University Wonju College of Medicine, Wonju, Korea
}

\begin{abstract}
Received August 30, 2019
Revised September 23, 2019

Accepted October 16, 2019
\end{abstract}

Address for correspondence
Woojae Han, PhD
Division of Speech Pathology and
Audiology, College of Natural
Sciences, Hallym University,
1 Hallymdaehak-gil,
Chuncheon 24252, Korea
Tel +82-33-248-2216
Fax +82-33-256-3420
E-mail woojaehan@hallym.ac.kr
Background and Objectives: The present study aimed to compare thresholds of direct bone-conduction (BC direct) with those of behaviorally measured $\mathrm{BC}$ pure-tone audiometry (PTA) and objectively measured BC auditory brainstem response (ABR) to confirm the clinical feasibility of their relationships. Subjects and Methods: Young adults with normal hearing participated in the study to determine the thresholds from three measurements at four testing frequencies. In the BC direct, the vibrator of a bone-anchored hearing aid softband was placed on the right mastoid of each subject. In both PTA and ABR, a B71 bone oscillator was placed on the subject's right mastoid. While the subject's thresholds of $\mathrm{BC}$ direct and BC PTA were determined with a clinically routine 5-dB step procedure, BC ABR was conducted to determine the individual's hearing sensitivity by a peak $V$ of the waveform using tone-burst and click stimuli. Results: The BC direct showed a different pattern between low and high frequencies. Precisely, its thresholds were 13.25 and $12.25 \mathrm{~dB} \mathrm{HL}$ at 0.5 and 1 $\mathrm{kHz}$, respectively, but 19 and $19.75 \mathrm{~dB} \mathrm{HL}$ at 2 and $4 \mathrm{kHz}$, respectively. A significant positive correlation existed between BC direct and PTA at $1 \mathrm{kHz}$, which was also correlated with ABR. Conclusions: Based on the current data, the thresholds of $B C$ direct were similar to BC PTA at low frequencies and BC ABR at high frequencies. The thresholds of $\mathrm{BC}$ direct might be predictable at approximately $5 \mathrm{~dB}$ higher (or lower) than that in PTA, although a large data set is required for standardization.

J Audiol Otol 2020;24(2):85-90

KEY WORDS: Bone-anchored hearing system · Direct bone-conduction Bone-conduction thresholds · Bone-conduction hearing.

\section{Introduction}

It is generally acknowledged that single-sided deafness, a profound sensorineural hearing loss in one ear with normal hearing in the other, can be treated with bone-anchored hearing systems (BAHSs) in the clinic [1]. Some patients with conductive or mixed hearing loss may also benefit from BAHSs, particularly those who have unilateral and/or bilateral hearing loss of at the most 50-60 dB HL and correct re-

This is an Open Access article distributed under the terms of the Creative Commons Attribution Non-Commercial License (https://creativecommons.org/licenses/by-nc/4.0/) which permits unrestricted non-commercial use, distribution, and reproduction in any medium, provided the original work is properly cited. sponses of $\geq 60 \%$ in speech recognition [2].

Currently, BAHSs have provided technical advantages to many patients in terms of better hygiene control and cosmetic concerns. For example, House and Kutz [3] suggested a new attachment technique via an external magnet rather than percutaneous abutment via a titanium screw, which increased the patient's risk of possible infection or skin overgrowth. Jang, et al. [4] confirmed the utility of a simple surgical procedure with a small incision and no risk of inner ear damage. Such technical advantages might provide a high satisfaction rate for users. Consequently, Flynn, et al. [5], who measured thresholds of patients with mixed hearing loss with BAHS implants, showed that the aided thresholds in patients were 
within the audible range for speech, indicating that BAHSs can offer as much significant benefit as conventional air-conduction hearing aids in terms of audibility and sound quality. While reporting a similar amount of threshold benefits as those by Flynn, et al., [5] Ricci, et al. [6] additionally confirmed improved scores in speech discrimination in patients with conductive and mixed hearing loss with BAHS implants.

Nevertheless, since the amount of amplification provided by the BAHS is based on the individual's frequency-specific sensorineural thresholds, reliable results (i.e., thresholds) from clinically objective measurements are necessary to initiate successful fitting for patients with BAHS implants. In detail, for the fitting procedure, clinicians need to determine the air-conduction and bone-conduction (BC) thresholds from the individual's audiogram. Then, unlike conventional hearing aids that are stimulated by air conduction, they should behaviorally measure direct $\mathrm{BC}$ (BC direct) using a software developed by the manufacturer to identify the best fit range. The measurement of BC direct includes sound attenuation through the skin and bone and needs to be considered for appropriate BAHS fitting. Precisely, the BC direct refers to a process of generation and transmission of signals that stem from the BAHS and thus allows direct measurement of the patient's hearing thresholds through a sound processor. If the amount of sound attenuation is not considered, the gain of the BAHS would be inadequate. Thus, the reliable thresholds obtained by BC direct measurement can support the accuracy of the initial BAHS fitting [7]. Moreover, it may be difficult to fit patients who have passive response, such as young children and/or older adults. As a solution to this problem, Rahne, et al. attempted objective measurements, such as auditory brainstem response (ABR) and cortical auditory evoked potentials (CAEP) in both individuals with normal hearing [8] and those with hearing impairment [9]. Their results suggested that CAEP measurement was the most objective and reliable tool to evaluate the BAHS fitting condition. However, a close look at their studies reveals that they had N1-P1-N2 responses only at 1 and $2 \mathrm{kHz}$ and included large inter- and intra-variance across the subjects (even in normal hearing). In addition to these limitations, cortical responses needed partial cooperation from the patients who were required to be awake during the measurement.

In contrast, pure-tone audiometry (PTA) is popularly used by clinicians because it is convenient and accurate and provides a comprehensive interpretation, while an $\mathrm{ABR}$ is an objective tool to confirm an individual's hearing condition and diagnose otoneural pathology. Both BC PTA and BC ABR have technical limitations in clinical application based on the maximum effective intensity level of approximately
$40 \mathrm{~dB}$ HL for BC stimuli. Furthermore, an appropriate amount of contralateral masking and high stimulus artifact as a result of electromagnetic energy from the bone vibrator during the measurement need to be considered [10]. However, several clinicians agree that the two measurements are important indicators in understanding cochlear sensitivity [11] and evaluating whether cochlea function is reserved. The present study aimed to compare the thresholds of $\mathrm{BC}$ direct with those of behaviorally measured BC PTA and objectively measured $\mathrm{BC}$ ABR, to which clinicians are fairly accustomed, and confirm the clinical feasibility of their relationships. The results will help clinicians understand $\mathrm{BC}$ direct measurement and determine the differences of the three BC measurements, even though they share the same auditory route, and will be a basis of BAHA fitting even in patients who do not provide an active psychometric response and thus have a difficulty in initiating BAHA fitting.

\section{Subjects and Methods}

\section{Participants}

Twenty young adults (10 male and 10 female) with normal hearing voluntarily participated in the study. The age ranged from 18 to 24 years [mean, 20.9 years; standard deviation (SD), 1.86]. All participants reported no history of head and neck abnormalities, ear surgery, otologic disease, and head trauma. They also underwent screening tests for participation in this study with a type-A tympanogram, thresholds of $\geq 15$ $\mathrm{dB} H \mathrm{HL}$ in each ear from 0.25 to $8 \mathrm{kHz}$, and air-bone gaps $<5$ $\mathrm{dB}$ HL. The experimental procedure was approved by the Institutional Review Board of Hallym University (HIRB-2019025). The participants provided written informed consent before initiation of the study.

\section{Experimental equipment and test stimuli}

In the $\mathrm{BC}$ direct measurement, the BAHA Fitting Software (version 5.3, Cochlear Ltd., Sydney, Australia) combined with the BAHA ${ }^{\circledR} 5$ Sound Processor (Cochlear Bone Anchored Solutions AB, Gothenburg, Sweden) was used. The stimuli were presented by a tone of $0.5,1,2$, and $4 \mathrm{kHz}$, which is a typical procedure to measure the thresholds in patients with BAHS implants [12]. The thresholds of BC PTA were behaviorally obtained using a B71 bone transducer headset (RadioEar, Middelfart, Denmark) connected to a GSI 61 audiometer (Grason-Stadler Inc., Eden Prairie, MN, USA) while being presented the same frequencies as those in $\mathrm{BC}$ direct.

Objectively, BC ABR was recorded with a Bio-logic Navigator Pro (Natus, Pleasanton, CA, USA). Its stimuli were 
presented using the same bone transducer as that in BC PTA (i.e., RadioEar B71 oscillator) as click and tone burst. In detail, the click stimuli with $100 \mathrm{~ms}$ in duration were presented at a rate of $11.1 / \mathrm{s}$ with alternating polarity. The tone-burst stimuli were presented at $0.5,1,2$, and $4 \mathrm{kHz}$ with alternating polarity. They were Blackman gated with two cyclesrise and fall times - and no plateau at a rate of $27.7 / \mathrm{s}$; consequently, the tone-burst stimuli at $0.5,1,2$, and $4 \mathrm{kHz}$ had a $1.5,2.0,3.0$, and $4.0 \mathrm{~ms}$ rise/fall time, respectively. For the time windows, $16 \mathrm{~ms}$ and $21.3 \mathrm{~ms}$ were applied for the click and tone-burst stimuli, respectively. Two stimuli were recorded using filter setting of 0.03 to $3 \mathrm{kHz}$ ( $5 \mathrm{~dB}$ /octave) and amplified using a gain of 100,000 . The samples $>23.8 \mu \mathrm{V}$ were rejected automatically. However, the maximum levels of stimuli differed depending on the type of stimuli and test frequencies due to high artifact: $50,60,70$, and $70 \mathrm{~dB} \mathrm{HL}$ for $0.5,1,2$, and $4 \mathrm{kHz}$ tone-burst and click stimuli, respectively. At each presentation level, 2,000 sweeps were averaged as minimum.

\section{Experimental procedures and conditions}

After conducting the hearing screening, the $\mathrm{BC}$ direct was measured in a quiet room. The vibrator of $\mathrm{BAHA}^{\circledR} 5$ softband (Cochlear Bone Anchored Solutions AB) was placed on the right mastoid of each subject to estimate the thresholds. When the signals were generated to the BAHA, the cochlea and/or osseo-integrated implant directly received the signal. The BC direct generated an amplified electromagnetic signal in response to sounds received from the sound processor. The amplified signal was transmitted to the internal device of the participant, and thus the signal caused the magnet to vibrate. Eventually, the vibrations were transmitted to the cochlea where the sounds became recognized [13]. While presenting $50 \mathrm{~dB}$ HL as an initial intensity level at each test frequency, the modified method of limits (also called the adaptive method of behavioral thresholds) using 5-dB step was applied to determine its thresholds [14].

Then, BC PTA was performed in a soundproof booth; the bone transducer was placed on the right mastoid of each subject to estimate the thresholds using the standard 5-dB adaptive method. During the BC ABR recording, the same bone vibrator was placed on the right mastoid. Click and toneburst stimuli were employed using a two-channel ipsilateral electrode montage with Fpz (ground), Fz (active), and left/ right ear lobe (reference). Electrode impedance in any pair of electrodes was maintained $<5 \mathrm{k} \Omega$. The data were rejected when artifacts exceeded $10 \%$ of the total sweep numbers. The stimuli intensity level was initially set to 50-70 dB HL, depending on the test frequency, and then reduced in $5-\mathrm{dB}$ steps until the individual threshold was confirmed. While labeling the wave $\mathrm{V}$ peak in the waveform, the thresholds were determined by two audiologists who had considerable experience in ABR recordings.

\section{Statistical analysis}

The statistical analysis was performed using two-way analysis of variance (ANOVA) with repeated measurement and Pearson correlation with SPSS software (version 21, IBM Corp., Armonk, NY, USA). In the ANOVA, the independent variables were the three measurements and their test frequencies, and the dependent variables were their thresholds. Pearson correlation was used to analyze any relationship among the thresholds of the three measurements. The criterion used for statistical significance was a $p$-value $<0.05$.

\section{Results}

Fig. 1 indicates the thresholds of BC PTA, BC direct, and $\mathrm{BC} \mathrm{ABR}$ in testing frequencies of $0.5,1,2$, and $4 \mathrm{kHz}$. Among the measurements, BC PTA showed the lowest thresholds across the frequencies whereas BC ABR had the highest thresholds. For example, four-frequency averages of the PTA and ABR thresholds were $3.81 \mathrm{~dB}$ HL (SD, 6.43) and $32.12 \mathrm{dBnHL}$ (SD, 13.99), respectively. However, BC direct provided a different pattern depending on the test frequency: lower thresholds for the low frequencies such as 500 and 1,000 Hz and higher thresholds for 2,000 and 4,000 Hz.

Based on the results of the ANOVA with repeated measurements, we confirmed a significant difference among the three measurements $[\mathrm{F}(2,38)=90.404, p<0.001]$. A Bonferroni post hoc test showed that the thresholds of BC PTA (mean, 3.81; SD, 1.01) were significantly lower than those of $\mathrm{BC}$ direct (mean, 16.37; SD, 1.27) $(p<0.001)$, which were significantly higher than those of BC ABR (mean, 32.12; $\mathrm{SD}, 2.11)(p<0.001)$. However, there was no significant difference in thresholds among the test frequencies $[\mathrm{F}(3,57)=0.818$, $p=0.489]$. The interaction between the three measurements and four test frequencies was revealed statistically $[\mathrm{F}(6,114)=$ 5.422, $p<0.001]$ because the thresholds of BC PTA were lower (or better) and $\mathrm{BC}$ direct results were increased (or worse) as the test frequency increased.

Interestingly, in the results of pairwise correlation among the three measurements and four test frequencies, a significantly positive correlation existed between BC PTA and BC direct $(\mathrm{r}=0.515, p=0.020)$ and between BC PTA and BC $\mathrm{ABR}$ at $1 \mathrm{kHz}(\mathrm{r}=0.445, p=0.049)$ (Table 1$)$. In more detail, we observed the individual's threshold of PTA for all subjects as a function of the $\mathrm{BC}$ direct thresholds (Fig. 2A) and 


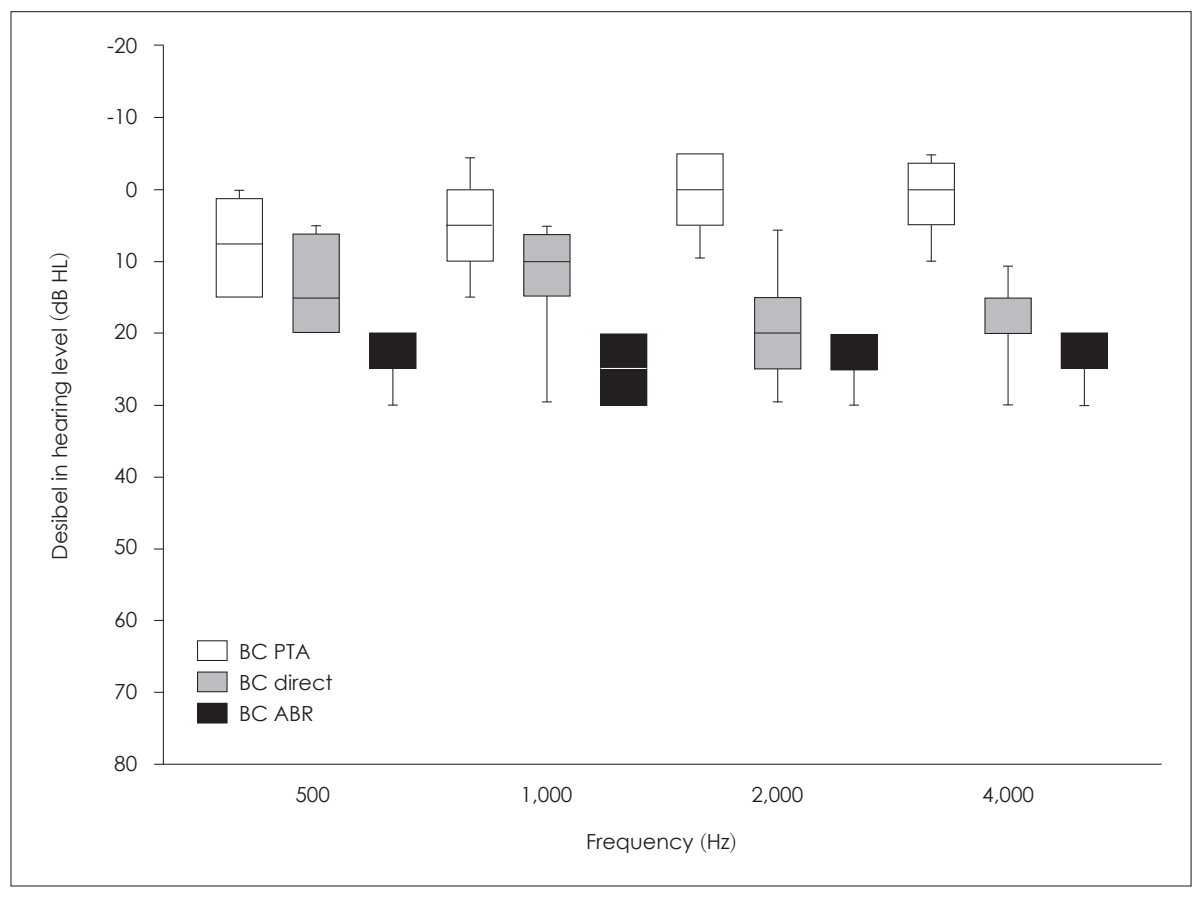

Fig. 1. Comparison of bone-conduction $(\mathrm{BC})$ thresholds among three measurements, pure-tone audiometry (PTA), BC direct, and auditory brainstem response (ABR), stimulated by frequency-specific tones at 0.5 , 1,2 , and $4 \mathrm{kHz}$. Noticeably, thresholds of the $\mathrm{BC}$ direct were closely located in BC ABR for 2 and $4 \mathrm{kHz}$.

Table 1. Pairwise correlations between bone-conduction thresholds of three measurements in four test frequencies

\begin{tabular}{|c|c|c|c|c|c|c|c|c|c|c|c|c|c|}
\hline \multirow{2}{*}{\multicolumn{2}{|c|}{ Variables }} & \multicolumn{3}{|c|}{$500 \mathrm{~Hz}$} & \multicolumn{3}{|c|}{$1,000 \mathrm{~Hz}$} & \multicolumn{3}{|c|}{$2,000 \mathrm{~Hz}$} & \multicolumn{3}{|c|}{$4,000 \mathrm{~Hz}$} \\
\hline & & \multirow{2}{*}{$\begin{array}{c}\text { PTA } \\
1.000\end{array}$} & \multirow[t]{2}{*}{ Direct } & \multirow[t]{2}{*}{ ABR } & \multirow[t]{2}{*}{ PTA } & \multirow[t]{2}{*}{ Direct } & \multirow[t]{2}{*}{$\mathrm{ABR}$} & \multirow[t]{2}{*}{ PTA } & \multirow[t]{2}{*}{ Direct } & \multirow[t]{2}{*}{$A B R$} & \multirow[t]{2}{*}{ PTA } & \multirow[t]{2}{*}{ Direct } & \multirow[t]{2}{*}{$A B R$} \\
\hline $500 \mathrm{~Hz}$ & PTA & & & & & & & & & & & & \\
\hline & Direct & 0.324 & 1.000 & & & & & & & & & & \\
\hline & $A B R$ & 0.088 & -0.051 & 1.000 & & & & & & & & & \\
\hline \multirow[t]{3}{*}{$1,000 \mathrm{~Hz}$} & PTA & $0.522^{*}$ & 0.241 & -0.278 & 1.000 & & & & & & & & \\
\hline & Direct & -0.116 & $0.618^{\dagger}$ & -0.235 & $0.515^{*}$ & 1.000 & & & & & & & \\
\hline & $A B R$ & 0.172 & -0.146 & -0.013 & $0.445^{*}$ & 0.204 & 1.000 & & & & & & \\
\hline \multirow[t]{3}{*}{$2,000 \mathrm{~Hz}$} & PTA & $0.479^{*}$ & 0.075 & -0.209 & $0.650^{\dagger}$ & 0.193 & $0.620^{\dagger}$ & 1.000 & & & & & \\
\hline & Direct & 0.079 & $0.461^{*}$ & -0.158 & 0.258 & $0.477^{\dagger}$ & 0.230 & 0.335 & 1.000 & & & & \\
\hline & $A B R$ & -0.354 & -0.394 & 0.221 & -0.360 & -0.290 & -0.100 & -0.071 & -0.332 & 1.000 & & & \\
\hline \multirow[t]{3}{*}{$4,000 \mathrm{~Hz}$} & PTA & 0.146 & -0.224 & -0.258 & 0.172 & -0.086 & 0.178 & 0.196 & 0.365 & -0.412 & 1.000 & & \\
\hline & Direct & -0.014 & 0.188 & -0.396 & -0.254 & -0.135 & -0.252 & 0.007 & 0.379 & -0.064 & 0.328 & 1.000 & \\
\hline & $A B R$ & -0.038 & -0.103 & 0.299 & -0.367 & -0.393 & -0.374 & -0.299 & -0.009 & 0.356 & -0.093 & 0.152 & 1.000 \\
\hline
\end{tabular}

$* p<0.05,{ }^{\dagger} p<0.01$. PTA: pure-tone audiometry, ABR: auditory brainstem response

that of $\mathrm{ABR}$ as a function of the PTA thresholds (Fig. 2B).

The threshold of the click-evoked BC ABR measurement was $31.25 \mathrm{dBnHL}(\mathrm{SD}, 10.24)$ for 20 subjects. However, no correlation was found between $\mathrm{BC}$ direct thresholds at $0.5,1$, 2 , and $4 \mathrm{kHz}$ and click-evoked ABR thresholds: $\mathrm{r}=0.199$, $p=0.401 ; \mathrm{r}=0.222, p=0.348 ; \mathrm{r}=0.284, p=0.225$; and $\mathrm{r}=-0.044$, $p=0.855$, respectively.

\section{Discussion}

While comparing the threshold of $\mathrm{BC}$ direct with those of behaviorally measured BC PTA and objectively measured
BC ABR for 20 subjects with normal hearing, the present study aimed to determine the reliability of the BC direct thresholds and confirm the clinical feasibility through their relationships for application to young or older patients who have passive behavioral responses.

As expected, the BC PTA showed the lowest thresholds across the frequencies, whereas the $\mathrm{BC} \mathrm{ABR}$ had the highest thresholds. Our results showed that the threshold difference of the four-tone average between BC PTA and BC ABR was approximately $28 \mathrm{~dB}$. This finding was supported by Muchnik, et al. [10] and Seo, et al. [15]. However, in previous studies, the average threshold of subjects with normal hearing 

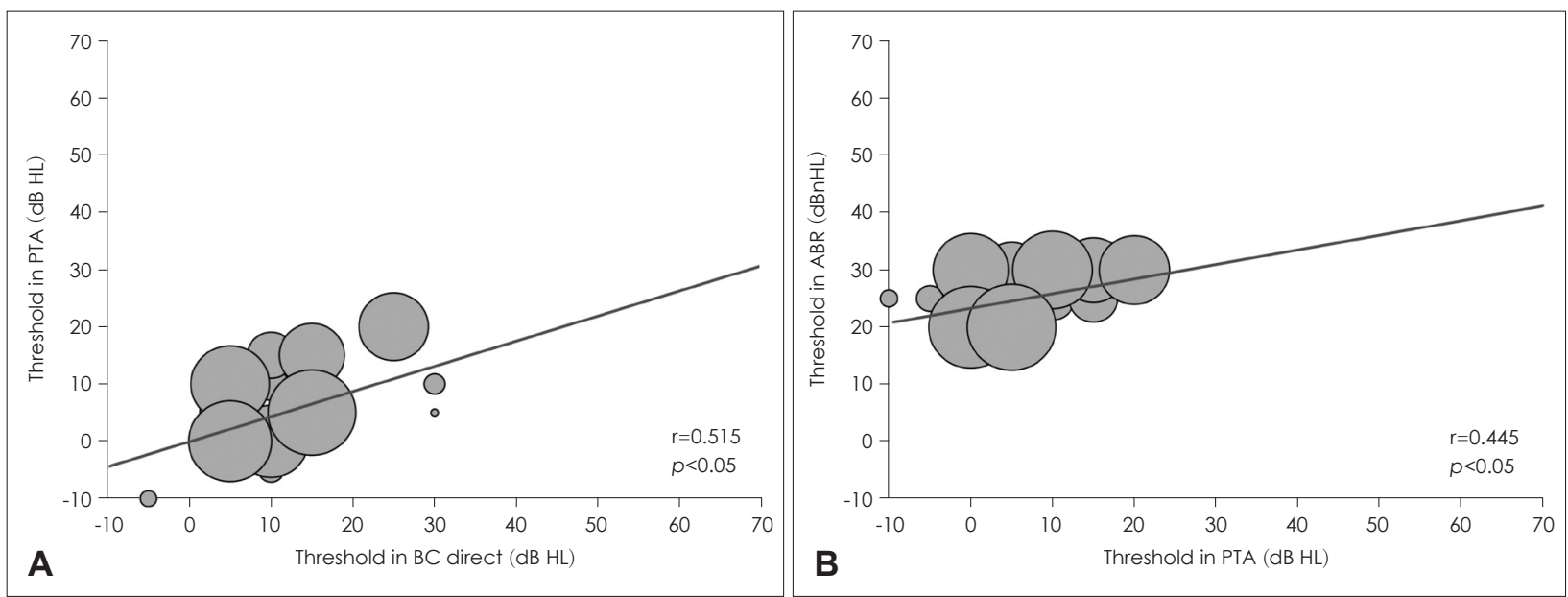

Fig. 2. Correlation graphs of an individual's threshold between pure-tone audiometry (PTA) and bone-conduction (BC) direct (A) and between PTA and auditory brainstem response (ABR) (B) at $1 \mathrm{kHz}$ shown as a bubble chart ( $\mathrm{n}=1$ for small to $n=4$ for large). Compared to a slope of Panel A, the Panel B showed lower slope which had a correlation between higher thresholds in ABR and lower thresholds in PTA.

was approximately $18-19 \mathrm{dBnHL}$ in the BC ABR measurement, and thus the threshold difference betwen BC PTA and BC ABR was $18 \mathrm{~dB}$, which indicates that our study had an approximately $10 \mathrm{~dB}$ higher threshold than those in previous studies on BC ABR. One possible reason for this discrepancy is the unpleasant pressure and pain caused by the bone vibrator while measuring the BC ABR thresholds, even with no difference in the experimental conditions.

One striking finding of the present study is that the $\mathrm{BC}$ direct provided the differentiated threshold pattern in terms of frequency. In other words, there were low (or better) thresholds in the low frequencies, such as 500 and $1,000 \mathrm{~Hz}$, and high (or worse) thresholds in the 2,000 and 4,000 Hz frequencies. However, as shown in Fig. 1, the group average of the $500 \mathrm{~Hz}$ thresholds was at least $5 \mathrm{~dB}$ worse than that of the $1,000 \mathrm{~Hz}$ thresholds. Hodgetts, et al. [16] explained this as inherent low frequency noise of the BAHA because the inherent noise masks the aided thresholds at 250 and $500 \mathrm{~Hz}$. This was also supported by Pfiffner, et al. [17] in that physical characteristics of the sound processor provided a maximal output force at approximately $1 \mathrm{kHz}$. If this statement extends to our correlation results, it would be a strong finding with a significantly positive correlation between PTA and $\mathrm{BC}$ direct and between PTA and ABR at $1 \mathrm{kHz}$. Christensen, et al. [18], who estimated the $\mathrm{BC}$ direct thresholds of the subjects with a BAHA softband, showed that BC direct thresholds were 6-14 dB higher than BC PTA thresholds, while supporting our results of $3.81 \mathrm{~dB}$ for BC PTA and $16.37 \mathrm{~dB}$ for $\mathrm{BC}$ direct. Additionally, their post hoc results were in accordance with our findings that the thresholds of $\mathrm{BC}$ direct at $1 \mathrm{kHz}$ were better than those at 500 or $4,000 \mathrm{~Hz}$. This is a possible result when considering the transmission characteristics of the ear [18].

Nevertheless, the BC direct thresholds seemed large up to $20 \mathrm{~dB}$ at four testing frequencies, although another study achieved similar results of a $17.5 \mathrm{~dB}$ maximum difference at $2,000 \mathrm{~Hz}$. This may be a possible limitation of the current study, which evaluated the individual with normal hearing with a BAHA softband. Therefore, we need to thoroughly investigate $\mathrm{BC}$ auditory pathways in a follow-up study. It is well known that the osseo-tympanic route (with sound radiated into the external ear canal, compression, and expansion of the petrous bone that results in displacement of fluid into the cochlea, and consequently basilar membrane motion) and the inertial effect of the middle ear ossicles and inner ear fluids constitute the most common measurements [19]. However, no studies have reported on the best dominant BC pathway in terms of force prorogation and loss. Therefore, the $\mathrm{BC}$ direct might be predictable at approximately $5 \mathrm{~dB}$ higher (or worse) than the BC PTA and 5-10 dB lower (or better) than the $\mathrm{BC}$ ABR in individuals with normal hearing, although a large data set is required for standardization. Further research should be conducted to gather data from patients with hearing impairment, including BAHA candidates/ users for comparison between normal and pathological difference of the $\mathrm{BC}$ route and thresholds.

\section{Acknowledgments}

This work was supported by the National Research Foundation of Korea grant (NRF 2019K1A3A1A47000527).

\section{Conflicts of interest}

The authors have no financial conflicts of interest.

\section{Author Contributions}

Conceptualization: Woojae Han. Data curation: Yeoju Kim and 
Sihun Park. Formal analysis: Sunghwa You and Chanbeom Kwak. Funding acquisition: Youngjoon Seo. Methodology: Sihun Park, Yeoju Kim, and Woojae Han. Project administration: Chanbeom Kwak. Supervision: Woojae Han. Validation: Youngjoon Seo and Jihyeon Lee. Visualization: Yeoju Kim and Sunghwa You. Writing - original draft: Yeoju Kim. Writing — review and editing: All authors.

\section{ORCID iDs}

Yeoju Kim

Woojae Han

Sihun Park

Sunghwa You

Chanbeom Kwak

Youngjoon Seo

Jihyeon Lee https://orcid.org/0000-0002-3358-5100

https://orcid.org/0000-0003-1623-9676

https://orcid.org/0000-0001-6085-2107

https://orcid.org/0000-0003-2458-4509

https://orcid.org/0000-0001-5657-7536

https://orcid.org/0000-0002-2839-4676

https://orcid.org/0000-0003-0896-9062

\section{REFERENCES}

1) Desmet J, Bouzegta R, Hofkens A, De Backer A, Lambrechts P, Wouters K, et al. Clinical need for a Baha trial in patients with single-sided sensorineural deafness. Analysis of a Baha database of 196 patients. Eur Arch Otorhinolaryngol 2012;269:799-805.

2) Hagr A. BAHA: bone-anchored hearing aid. Int J Health Sci (Qassim) 2007;1:265-76.

3) House JW, Kutz JW Jr. Bone-anchored hearing aids: incidence and management of postoperative complications. Otol Neurotol 2007;28: 213-7.

4) Jang DS, Shin DH, Han W, Kong TH, Seo YJ. Baha attract implantation using a small incision: initial report of surgical technique and surveillance. Clin Exp Otorhinolaryngol 2019 Jul 6 [Epub]. https// doi.org/10.21053/ceo.2019.00381.

5) Flynn MC, Sadeghi A, Halvarsson G. Baha solutions for patients with severe mixed hearing loss. Cochlear Implants Int 2009;10(Sup 1):43-7.

6) Ricci G, Della Volpe A, Faralli M, Longari F, Gullà M, Mansi N, et al. Results and complications of the Baha system (bone-anchored hearing aid). Eur Arch Otorhinolaryngol 2010;267:1539-45.

7) Flynn MC, Hillbratt M. Improving the accuracy of Baha(R) fittings

through measures of direct bone conduction. Clin Exp Otorhinolaryngol 2012;5(Suppl 1):S43-47.

8) Rahne T, Ehelebe T, Rasinski C, Götze G. Auditory brainstem and cortical potentials following bone-anchored hearing aid stimulation. J Neurosci Methods 2010;193:300-6.

9) Rahne T, Ehelebe T. Objective estimation of frequency-specific pure-tone hearing thresholds following bone-conduction hearing aid stimulation. Scientific World Journal 2014;2014:247942.

10) Muchnik C, Neeman RK, Hildesheimer M. Auditory brainstem response to bone-conducted clicks in adults and infants with normal hearing and conductive hearing loss. Scand Audiol 1995;24:185-91.

11) Sheykholeslami K, Mohammad HK, Sébastein S, Kaga K. Binaural interaction of bone-conduction auditory brainstem responses in children with congenital atresia of the external auditory canal. Int J Pediatr Otorhinolaryngol 2003;67:1083-90.

12) Flynn MC, Sadeghi A, Halvarsson G. Benefits of directional microphones and noise reduction circuits for improving $B a h a(R)$ hearing performance. Cochlear Implants Int 2011;12 Suppl 1:S139-41.

13) Hough JV, Richard GL, Barton Jr KE, Dicarlo P, Chow RY. Direct bone conduction hearing aid device [Internet]. U.S. Patent and Trademark office; 1986 Sep 23; No. 4,612,915 [cited 2019 Jul 30]. Available from: URL: https://patents.google.com/patent/US4612915A/en.

14) Han J, Cho SH, Cho SJ, Gu HR. A comparison between AC. BC auditory steady-state response thresholds and pure tone thresholds in the modulation frequency of $40 \mathrm{~Hz}$ and $80 \mathrm{~Hz}$. Audiol 2005;1:40-3.

15) Seo YJ, Kwak CB, Kim SA, Park YA, Park KH, Han WJ. Update on bone-conduction auditory brainstem responses: a review. J Audiol Otol 2018;22:53-8.

16) Hodgetts WE, Håkansson BE, Hagler P, Soli S. A comparison of three approaches to verifying aided Baha output. Int J Audiol 2010; 49:286-95.

17) Pfiffner F, Caversaccio MD, Kompis M. Audiological results with $\mathrm{Baha}(\mathrm{R})$ in conductive and mixed hearing loss. In: Implantable bone conduction hearing aids (eds. Kompis M, Caversaccio MD), Vol. 71. Basel: Karger Publishers;2011. p.73-83.

18) Christensen L, Smith-Olinde L, Kimberlain J, Richter GT, Dornhoffer JL. Comparison of traditional bone-conduction hearing aids with the Baha system. J Am Acad Audiol 2010;21:267-73.

19) Türkman T, Kaygusuz İ, Başar F, Karkıdağ T, Keleş E, Birkent ÖF, et al. Normalization of bone conduction auditory brainstem evoked responses in normal hearing individuals. J Int Adv Otol 2018;14:418-25. 